\title{
Nimesulide induced Stevens- Johnson Syndrome
}

\author{
Karn $D^{1}$, Khatri $R^{2}$
}

\author{
${ }^{1}$ Lecturer, Department of Dermatology and \\ Venereology, Dhulikhel Hospital , KUTH, \\ Dhulikhel, Kavre \\ ${ }^{2}$ Inter Doctor, Dhulikhel Hospital, KUTH.
}

\author{
Correspondence \\ Dr. Dharmendra Karn \\ Department of Dermatology and Venereology, \\ Dhulikhel Hospital, KUTH, \\ Dhulikhel, Kavre, \\ e-mail : dddkarn@gmail.com
}

\begin{abstract}
Stevens - Johnson syndrome (SJS) is a severe, episodic, acute mucocutaneous reaction that is most often elicited by drugs and occasionally by infections. We report a case of 29 years old woman who developed Stevens - Johnson syndrome following the ingestion of Nimesulide tablets. Nimesulide is a nonsteroidal anti-inflammatory (NSAID) drug with antipyretic and analgesic properties. To the best of our knowledge there have been no previous reports of SJS induced by the ingestion of Nimesulide in the medical literature. This case highlights the importance of Nimesulide and other NSAIDS as the possible cause of Stevens - Johnson syndrome which needs to be borne in mind by the treating physician in cases of drug reactions.
\end{abstract}

\section{Key words}

Nimesulide, Stevens - Johnson syndrome.

\section{Introduction}

Stevens- Johnson Syndrome, first described in 1922, is a serious mucocutaneous illness with systemic symptoms characterized by the presence of flat, atypical target lesions and the epidermal detachment is $<10 \%$ of the total body surface area (BSA) ${ }^{1}$. Two or more mucosal sites are usually affected. Recent reports have linked SJS to the use of drugs rather than other etiologic factors. Antibiotics e.g. sulphonamides are the most common cause of SJS, followed by analgesics, cough and cold medication, nonsteroidal anti-inflammatory drug (NSAID), psycho-epileptics, and antigout drugs ${ }^{2}$.

\section{Case Report}

A 29 yrs old lady presented to emergency department of our hospital with complaints of fever for five days not associated with chills and rigors with no diurnal variations, swelling of face and lips for 5 days and rashes over whole body for 4 days. Rashes initially started over face which spread to the neck, chest, back, and abdomen, upper and lower legs within 24 hours. She also gave history of burning micturation for the same duration.
On examination, she was ill looking; her vitals were stable except for pulse of $124 / \mathrm{min}$. Ocular examination revealed congestion of both conjunctiva with mucoid discharge and matted eyelashes. Oral examination revealed oedematous lips with decreased mouth opening, crusting of both lips with swollen tongue and thick mucoid discharge (Fig. 1). Erythematous maculopapular rashes were present over face, neck, chest, back, abdomen, upper and lower limbs including genitalia with confluence of rashes over abdomen, and lower limbs (Fig. 2). Few atypical target lesions were present over lower limbs (Fig. 3). Large ulcer was present over the genitalia. Other systemic examinations were within normal limits.

On further detailed inquiry, she gave the history of ingestion of Nimesulide tablets as the over the counter drug for mild headache five days ago, she then developed swelling of lips immediately after the drug ingestion followed by fever and rashes.

Haematological and biochemical investigations were sent, which revealed slight derangement in the Liver Function Test with SGPT 291 IU/L, SGOT of 262 IU/L and ALP of $126 \mathrm{IU} / \mathrm{L}$. Bilirubin levels, Renal Function Tests, Chest X-Ray , Urine RME all were within normal limits. 
With the history, clinical examination and investigatory findings diagnosis of Nimesulide induced Stevens Johnson Syndrome was made and the patient managed with parenteral steroids, third generation cephalosporins, and local application of steroid intraorally followed by mouth paint. We also carried out the causality assessment as per the Naranjo Score which revealed the association as 'probably' associated with the drug.

The patient's condition dramatically improved after the initiation of treatment and the patient, on steroids, was discharged on fifth day of admission on regular follow up.

\section{Discussion}

Erythema Multiforme group of diseases include a number of disease conditions characterized by acute self limiting exanthematic intolerance reactions that share at least two characteristic features- target lesions ( stable circular erythemas or urticarial plaques with areas of blistering, necrosis and/or resolution in concentric array ) and, histologically, satellite cells ( or more widespread) necrosis of the epidermis ${ }^{3}$.

No generally accepted classification still exists for these disease conditions. More recently, there has been a tendency to consider erythema multiforme major and minor as a part of spectrum, often related to infections (mostly Herpes Simplex Virus) and to separate SJS and Toxic Epidermal Necrolysis (TEN), both of which are more closely linked to drug sensitivities, which may be regarded as severe variants of more severe disease ${ }^{4}$.

SJS and TEN are both characterized by rapidly expanding, often irregular macules ("atypical target lesions") and involvement of more than one mucosal site (oral, conjunctival, and anogenital). They are separated $y$ the severity of disease condition in the form of epidermal detachment, SJS being involvement of less than ten percent of the Body Surface Area ( BSA), TEN more than thirty and "SJS-TEN overlap" if in between.

Although SJS-TEN has a poletiologic reaction pattern, drugs are clearly the causative factor ( 80 to 95 percent of patients with TEN and, more than 50 percent of those with SJS) and only a minority are linked with infection, vaccination, or graft-versus host disease and a very small number idiopathic.

The list of causative drugs may vary from country to country and over time because of differences in drug use, three groups are cited as the most common triggers in all surveys and reviews; antibacterial sulfonamides, anticonvulsants, and nonsteroidal anti-inflammatory drugs (NSAIDS). Antimalarials, allopurinol and a few follow close second.
Among the NSAIDS, especially pyrazolon derivatives e.g. Phenylbutazone and oxicam derivatives are more linked with these drug reactions. Nimesulide in the other hand with lesser GI adverse effects than compared to its counterparts of its family, though rare has been attributed to renal disorders, allergic reactions, asthma, and hematological reactions. In the mid year of 2007 it had been banned from certain European nations due to its adverse effects on the liver, which later was proven wrong by the Committee for Medicinal Products for Human Use (CHMP) stating that benefits of Nimesulide outweighs its adverse effects. ${ }^{5-6}$

Till date no SJS has been reported to occur due to the ingestion of Nimesulide tablets in any medical literature to the best of our knowledge. The easy availability of this drug as an over the counter medication for any form of headache, myalgias, arthralgias in developing nation like ours, care should be taken by the treating physician of its possible reaction while prescribing the drug and to regard it as the possible cause for the drug reactions that follow in a patient especially if the etiology is regarded as unknown till then.

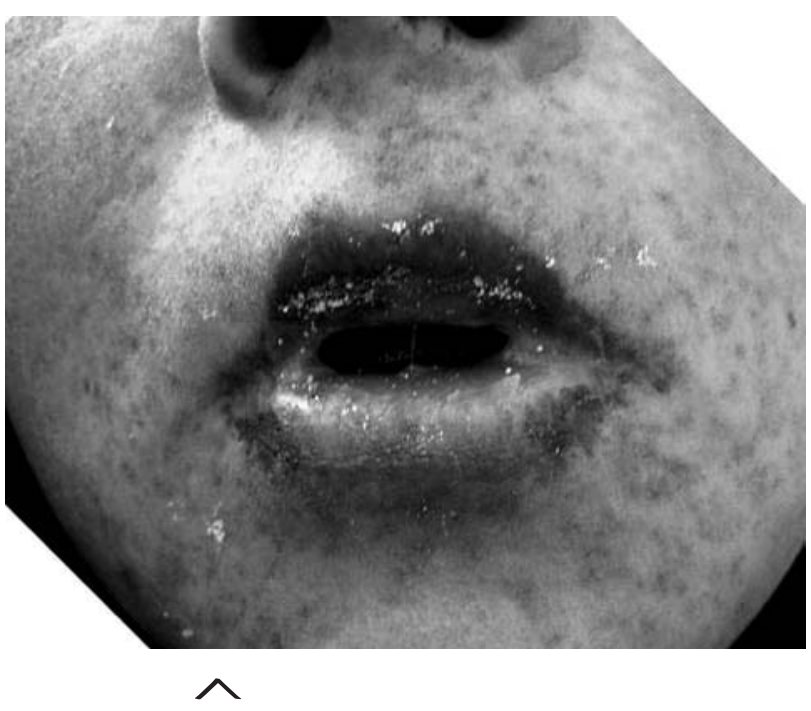

Fig. 1 Note the crusting of lips, adhesions, decreased mouth opening and discharges present 


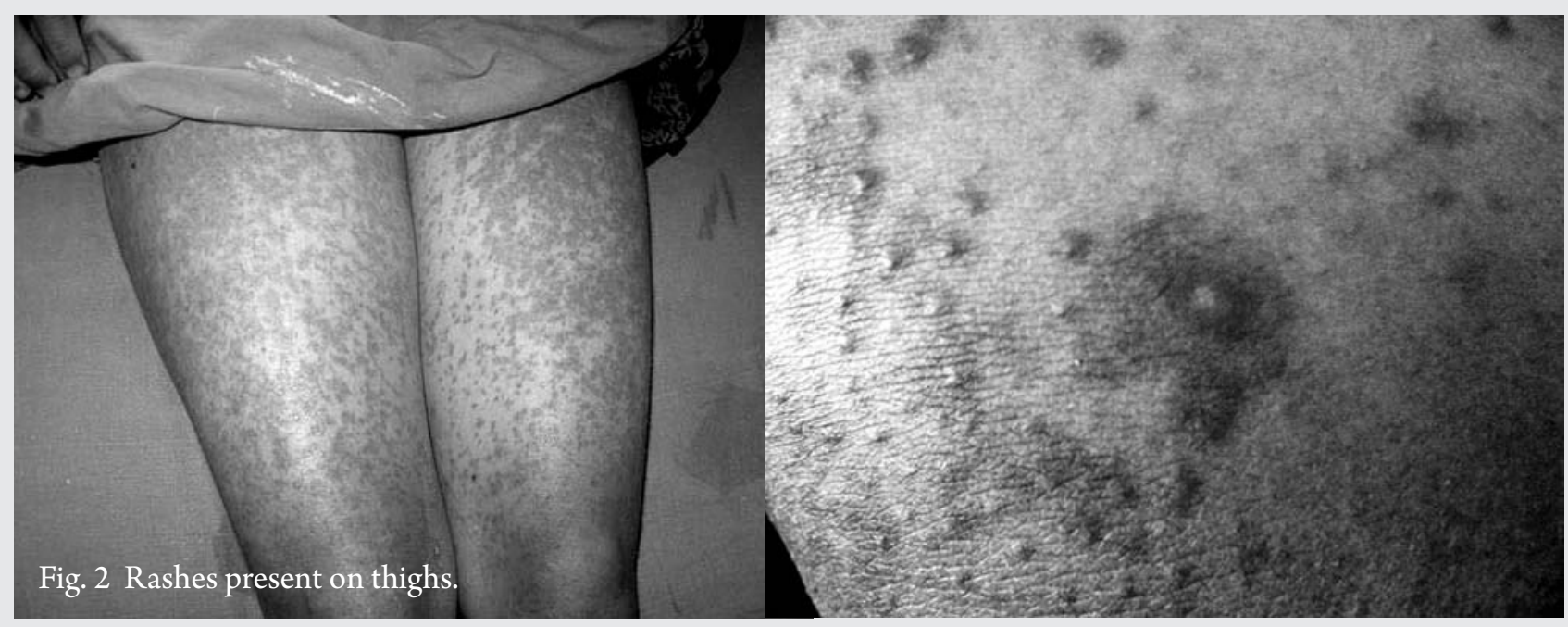

\section{References}

Fig. 3 A typical Target Lesion

1. Stevens AH, Johnson FC. A new eruptive fever associated with stomatitis and ophthalmia: report of two cases in children. Am J Dis Child 1922; 24: 526-7.

2. Fagot JP, Mockenhaupt M, Bowes-Bavinck JN, et al 2001Nevirapine and the risk of Stevens - Johnson syndrome or toxic epidermal necrolysis. AIDS 2001, 15:1843-1848.

3. Peter O. Fritsch, Ramon Ruiz-Maldonado; Erythema Multiforme, Stevens-Johnson Syndrome, and Toxic Epidermal Necrolysis ; Fitzpatrick's DERMATOLOGY in General Medicine; Vol 1. Sixth Edition, The McGraw Hill, 2003, 543- 556.

4. S.M. Breathnach, Erythema Multiforme, StevensJohnson Syndrome, and Toxic Epidermal Necrolysis. Rook's Textbook of Dermatology. Vol.4, Seventh Edition. Blackwell Publishing,2004: 74.1-74.19.

5. Information assessed from http://emea.europea.eu/ pdfs/general/direct/pr/43260407/en.pdf. As on 08/11/09.

6. Information assessed from http://niesulide-update. com/index.php?option=com_context\&task=veiw\&id. As on 08/11/09 